\section{Mastite lactacional e a iniciativa Hospital Amigo da Criança, Feira de Santana, Bahia, Brasil}

\author{
Lactational mastitis and Baby-Friendly Hospital \\ Iniciative, Feira de Santana, Bahia, Brazil
}

\author{
1 Universidade Estadual \\ de Feira de Santana \\ Feira de Santana, Brasil. \\ 2 Universidade Federal \\ da Bahia, Salvador, Brasil. \\ 3 Faculdade de Medicina \\ de São José do Rio Preto. \\ São José do Rio Preto, Brasil. \\ Correspondência \\ G. O. Vieira \\ Universidade Estadual \\ de Feira de Santana. \\ $A v$. Universitária $s / n$ \\ Feira de Santana, $B A$ \\ 44031-060, Brasil. \\ graciete.vieira@terra.com.br
}

\begin{abstract}
This study aimed to evaluate whether follow-up of breastfeeding mothers at maternity hospitals classified as "Baby-Friendly Hospitals" in Feira de Santana, Bahia, Brazil, was a protective factor against mastitis. This was a cross-sectional study in which 2,543 mothers of infants $(<1$ year of age) were selected by simple stratification and interviewed by 104 college students at immunization services. Measures of association (odds ratio, 95\%CI) and statistical significance were calculated by the chi-square test and respective $p$ values. Multivariate analysis was performed using the logistic regression model. Women who delivered in "Baby-Friendly Hospitals" had a lower prevalence of lactational mastitis (3.6\% vs. 5.3\%; OR = 0.68; 95\%CI: 0.46-1.01). Additionally, delivery in Baby-Friendly Hospitals $(O R=0.71 ; 95 \% C I: 0.48-1.06)$, absence of nipple fissure (OR =0.27; 95\%CI: 0.19-0.40), and no maternal outdoor work $(O R=0.64 ; 95 \% C I$ : 0.44-0.94) were also associated with a decreased prevalence of lactational mastitis. Prevalence of lactational mastitis was observed to be lower in women delivering in "Baby-Friendly Hospitals" with breastfeeding counseling programs.
\end{abstract}

Breast Feeding; Mastitis; Program Evaluation; Hospital Practice
Graciete Oliveira Vieira 1,2 Luciana Rodrigues Silva ${ }^{2}$ Carlos Maurício Cardeal Mendes 2 Tatiana de Oliveira Vieira ${ }^{3}$

\section{Introdução}

Para a World Health Organization (WHO), a mastite lactacional é uma importante causa de desmame 1 , chegando a ser a terceira razão informada pelas mães 2 . Por ser um processo doloroso, interfere na qualidade de vida da mulher-mãe e, conseqüentemente, na relação mãefilho. A mastite é um processo inflamatório da mama, que pode ser acompanhado ou não de infecção, com uma incidência relatada entre 2,6 a 33\%, numa revisão realizada pela WHO 1 . Ademais, tem sido relatado maior risco de transmissão do vírus HIV nessa afecção 3,4,5,6.

Recentemente, tem sido também diagnosticada uma condição definida como mastite subclínica, nos casos de aumento da razão do sódio-potássio no leite, acompanhada de aumento de células inflamatórias, citoquinas e interleucinas-8, na ausência de sintomas clínicos de mastite $1,4,6,7,8$.

A mastite tem início, geralmente, na segunda ou terceira semana do puerpério, podendo ocorrer, no entanto, em qualquer estágio da lactação 1 e, quando não tratada, pode evoluir para abscesso 9,10. Quando há infecção, ocorrem manifestações sistêmicas importantes, como febre alta, calafrios e mal-estar. No exame físico, a parte afetada da mama apresenta-se vermelha, quente, edemaciada e dolorida. A mastite pode ocorrer, na maioria das vezes, unilateralmente, em área localizada ou em vários pontos da mama 1. 
As principais causas da mastite são a estase lática, causada por uma remoção ineficiente do leite e infecção 1, com uma maior prevalência do Staphylococcus aureus como agente infeccioso em até 50 a $60 \%$ dos casos, dentre outros microorganismos 11. Vários fatores como idade, primiparidade, estresse, fadiga, nível de escolaridade da mãe, trabalho fora do lar, trauma mamilar 1,12,13, má pega 14, fissura mamilar 1,12,15,16 e ingurgitamento mamário têm sido relatados como agravantes para o risco de mastite 17 .

A mastite lactacional pode ser evitada através de medidas que impeçam a instalação da estase lática, tais como: a boa pega, o aleitamento sob livre demanda, o esvaziamento completo da mama durante a amamentação, a ordenha do peito nos casos de produção de leite maior que a demanda do lactente e, também, o estímulo ao aleitamento materno e ao autocuidado.

É importante lembrar que as ações preventivas da mastite estão incorporadas às rotinas da Iniciativa Hospital Amigo da Criança (IHAC), que tem como propósito realizar um trabalho de promoção, incentivo e apoio ao aleitamento materno, através de modificações de rotinas hospitalares no pré-natal, sala de parto, alojamento conjunto, berçário e formação de grupos de apoio à amamentação após a alta hospitalar 18. No Estado da Bahia, existem oito hospitais credenciados como amigos da criança, sendo que dois desses estão localizados na Cidade de Feira de Santana.

O presente estudo tem como objetivo investigar se o acompanhamento da nutriz, com ênfase na importância da amamentação, e o seu preparo para lidar com a lactação, proposto pelos hospitais Amigos da Criança, mostraram-se fatores de proteção contra a mastite lactacional, nas mulheres atendidas nos hospitais com o programa, no Município de Feira de Santana. Foi também estudada a ocorrência da mastite segundo algumas características maternas, das crianças e em relação ao manejo da lactação.

\section{Metodologia}

Trata-se de um estudo de base populacional do tipo transversal, realizado na Cidade de Feira de Santana, situada a $108 \mathrm{~km}$ da capital do Estado - Salvador, Brasil - com uma população em torno de 480.949 habitantes 19. Os dados foram coletados durante a segunda etapa da Campanha Nacional de Vacinação, dia 25 de agosto de 2001.

\section{População do estudo}

A população alvo, da qual se obteve a amostra, foi constituída pelas crianças menores de um ano, que haviam completado a idade até o dia do inquérito, procedentes da área urbana da Cidade de Feira de Santana. A estimativa da população alvo teve como base os dados fornecidos pela Secretaria de Saúde do município sobre o número de crianças menores de um ano, vacinadas em cada unidade, durante a primeira etapa de vacinação do mesmo ano. A unidade de análise correspondeu às mães das crianças menores de um ano, selecionadas para a amostra.

\section{Desenho amostral}

O plano amostral foi probabilístico e estratificado. No planejamento da cobertura vacinal do município, a Secretaria de Saúde dividiu a cidade em quatro "comandos" (estratos), áreas topográficas representadas por bairros contíguos, sendo também levados em consideração a organização e o número de crianças vacinadas na primeira etapa da campanha do mesmo ano. No dia da coleta de dados, a vacinação ocorreu em 62 unidades - postos de saúde e escolas - localizados nos bairros componentes de cada estrato. Uma amostra foi retirada de cada estrato. Foram sorteadas, respectivamente, 12 das 17 unidades de vacinação do primeiro e segundo estratos, e 10, dentre as 14 unidades existentes, no terceiro e quarto estratos. $\mathrm{O}$ questionário foi aplicado a todas as mães das crianças menores de um ano, que compareceram às 44 unidades selecionadas, para compor a amostra. Responderam ao questionário 2.543 mães; essa parcela correspondeu a $26,4 \%$ da população de crianças menores de um ano, residentes no Município de Feira de Santana (9.615), no ano 200120 . Apenas quatro mães se recusaram a participar da pesquisa, taxa considerada insignificante.

\section{Instrumento e coleta de dados}

A pesquisa foi realizada por 104 universitários da área de saúde, previamente treinados. As mães eram abordadas, na fila de vacinação, por dois universitários, que informavam que estavam realizando um inquérito sobre alimentação infantil, e, após o consentimento livre e esclarecido, os questionários eram aplicados individualmente. O plano piloto foi realizado na primeira etapa de vacinação, com aplicação de 167 questionários por dez inquiridores. 


\section{Variáveis}

Definiu-se como variável independente principal a implantação da IHAC, e referiu-se como dependente a ocorrência da mastite lactacional. Foi considerado como fator de proteção o atendimento, durante o parto, nos hospitais com IHAC. As características da população do estudo, que poderiam interferir sobre a associação principal, avaliadas em relação às mães, foram: escolaridade, idade, paridade e trabalho fora do lar, sendo consideradas como fatores de proteção, escolaridade maior que o nível básico do Ensino Fundamental, não ser primípara, ter idade maior ou igual a vinte anos e não se ausentar do lar para trabalhar. Com relação à criança, foi investigado o peso ao nascer e o uso de chupetas, sendo considerados como fatores de proteção ter peso, ao nascer, maior ou igual a $2.500 \mathrm{~g}$ e não usar chupetas. Quanto à conduta durante a lactação, foram investigados a orientação do aleitamento materno no pré-natal e o alojamento conjunto; a orientação quanto à ordenha no alojamento conjunto; a referência de fissura de mamilo e, por fim, o tipo de aleitamento materno. Foram analisados como fatores de proteção: mães em aleitamento, orientação quanto ao manejo da amamentação e ausência de fissura mamilar.

Foram consideradas como amamentadas todas as crianças que fizeram uso de leite de peito, independente do uso de outros alimentos. Em aleitamento exclusivo, estavam aquelas alimentadas somente com leite materno. Em relação à mastite, foi questionado à mãe quanto a sua ocorrência, e considerados, para análise, os eventos que haviam sido confirmados por médico ou enfermeiro, segundo a informação das mães. Quanto à fissura mamilar, foi perguntado às nutrizes se elas, durante a lactação, tiveram fissura (rachadura no bico do peito). O nome do hospital em que a criança nasceu e o peso de nascimento foram verificados no Cartão da Criança.

\section{Análise}

Foram calculadas medidas de associação (razão de prevalência, com intervalo de confiança a 95\%) e de significância estatística, através do teste do qui-quadrado com respectivo valor de p. Foram descritas as características da população de estudo, de acordo com a ocorrência ou não da mastite, apresentando a distribuição das variáveis entre os expostos e não-expostos, sendo considerados como significantes valores $\leq 5 \%$. Em seguida, realizou-se análise multivariada através do modelo de regressão logística.
Na regressão logística, inicialmente, foi testada a associação de cada variável independente com a variável dependente (mastite), sendo selecionadas, para a etapa subseqüente, aquelas que obtiveram valores de $\mathrm{p}$ no teste da razão da verossimilhança menores ou iguais a $25 \%$. A segunda etapa consistiu em construir um modelo com as variáveis pré-selecionadas na fase anterior, utilizando o método de seleção de trás para frente, backward, e nível de significância de 0,17. A terceira etapa foi destinada a testar as possíveis interações existentes entre a variável independente principal (IHAC) e cada uma das demais variáveis independentes mantidas no modelo até aquela etapa. Na fase final, as variáveis mantidas na segunda etapa, juntamente com os possíveis termos de interação pré-selecionados, foram colocadas conjuntamente no modelo, mais uma vez, utilizando-se o método de trás para frente e determinandose, então, os coeficientes de regressão logística, as razões de chances e seus intervalos de confiança a 95\%. O ajuste do modelo foi verificado pelo teste de Hosmer-Lameshow.

Por tratar-se de um estudo transversal, foram calculadas as estimativas das razões de prevalências e seus respectivos intervalos de confiança, correspondentes às razões de chances obtidas na regressão logística 21 . Os pacotes estatísticos utilizados foram o SPSS for Windows 10.0 (SPSS Inc., Chicago, Estados Unidos) e o STATA 7.0 (Stata Corporation, College Station, Estados Unidos). A pesquisa foi aprovada pela Comissão de Ética e Pesquisa da Universidade Federal da Bahia.

\section{Resultados}

Na Tabela 1, são apresentados dados relativos às características da população do estudo, conforme a presença ou ausência da mastite. Foi observada uma associação no sentido da proteção relativa à mastite, quando a lactante não se ausentava do lar para trabalhar. As características maternas, quanto à idade, escolaridade e paridade, não apresentaram diferenças significantes de prevalências entre os expostos e não-expostos. Do mesmo modo, em relação às crianças, as variáveis peso ao nascer e uso de chupetas não se associaram, de modo significante, à mastite (Tabela 1).

$\mathrm{Na}$ análise das variáveis pesquisadas, quanto ao manejo da lactação, conforme a presença ou ausência da mastite lactacional, apresentadas na Tabela 2, notou-se prevalência menor dessa patologia entre as mulheres que pariram nos hospitais com IHAC, quando comparadas 
Características da população de estudo de acordo com a presença ou ausência de mastite.

\begin{tabular}{|c|c|c|c|c|c|c|}
\hline \multirow[t]{3}{*}{ Variáveis } & & \multicolumn{5}{|c|}{ Mastite } \\
\hline & \multicolumn{2}{|c|}{ Sim } & \multicolumn{2}{|c|}{ Não } & \multirow[t]{2}{*}{ RP (IC95\%) } & \multirow[t]{2}{*}{$p$} \\
\hline & $\mathrm{n}$ & $\%$ & $\mathrm{n}$ & $\%$ & & \\
\hline \multicolumn{7}{|l|}{ Idade materna (anos) } \\
\hline$<20$ & 24 & 4,1 & 558 & 95,9 & $1,19(0,77-1,84)$ & 0,441 \\
\hline$\geq 20$ & 96 & 4,9 & 1.865 & 95,1 & & \\
\hline \multicolumn{7}{|l|}{ Escolaridade materna } \\
\hline$\leq$ básico fundamental & 33 & 4,6 & 692 & 95,4 & $1,05(0,71-1,50)$ & 0,802 \\
\hline > básico fundamental & 87 & 4,8 & 1.731 & 95,2 & & \\
\hline \multicolumn{7}{|l|}{ Paridade } \\
\hline Primípara & 57 & 4,8 & 1.123 & 95,2 & $0,96(0,68-1,36)$ & 0,805 \\
\hline Multípara & 63 & 4,6 & 1.300 & 95,4 & & \\
\hline \multicolumn{7}{|l|}{ Peso ao nascer (gramas) } \\
\hline$<2.500$ & 10 & 5,2 & 181 & 94,8 & $0,90(0,60-2,10)$ & 0,730 \\
\hline$\geq 2.500$ & 110 & 4,7 & 2.242 & 95,3 & & \\
\hline \multicolumn{7}{|l|}{ Uso de chupetas } \\
\hline Sim & 70 & 4,8 & 1.385 & 95,2 & $0,95(0,67-1,36)$ & 0,800 \\
\hline Não & 50 & 4,6 & 1.038 & 95,4 & & \\
\hline \multicolumn{7}{|l|}{ Trabalho fora do lar } \\
\hline Sim & 39 & 6,5 & 557 & 93,5 & $0,64(0,44-0,92)$ & 0,020 \\
\hline Não & 81 & 4,2 & 1.866 & 95,8 & & \\
\hline
\end{tabular}

àquelas que pariram em hospitais sem o programa. Foi observada, também, uma associação no sentido da proteção relativa à mastite, quando a lactante teve orientação de aleitamento materno no pré-natal e alojamento conjunto (Tabela 2). A ocorrência de mastite foi significativamente menor dentre as mães que amamentavam de modo exclusivo ou não, quando comparadas àquelas que desmamaram. Por sua vez, a mastite lactacional foi significantemente mais prevalente dentre as mães que apresentaram fissura de mamilos (Tabela 2). No entanto, o ensinamento da técnica da ordenha antes da alta hospitalar não apresentou diferenças significantes de prevalências entre os expostos e não-expostos (Tabela 2).

Os resultados da análise da regressão logística, apresentados na Tabela 3, mostram as variáveis que permaneceram no modelo final. As variáveis identificadas como fatores de proteção contra a mastite foram: ser atendida nos serviços com IHAC (RP = 0,71; IC95\%: 0,48$1,06)$, ausência de fissura mamilar $(\mathrm{RP}=0,27$; IC95\%: 0,19-0,40) e não se ausentar do lar para trabalhar $(\mathrm{RP}=0,64$; IC95\%: 0,44-0,94).
Não foi identificada nenhuma interação entre a variável independente principal (IHAC) e as demais variáveis independentes pesquisadas. O procedimento de diagnóstico do ajuste do modelo mostrou um bom ajuste do modelo aos dados analisados.

\section{Discussão}

No presente estudo, observou-se que as lactantes que pariram nos hospitais com IHAC estiveram associadas, a menor prevalência de mastite lactacional. Embora o valor do teste de significância tenha correspondido à probabilidade do acaso maior do que o nível de significância definido para o estudo, o resultado obtido pode ser considerado limítrofe e sugere associação no sentido da proteção contra a mastite lactacional.

O tema mastite é pouco pesquisado em todo o mundo, e trabalhos publicados são, muitas vezes, retrospectivos e com amostras não representativas; grande número desses trabalhos foi realizado em animais. Em revisão re- 
Variáveis referentes ao manejo da lactação da população de estudo segundo a presença ou ausência de mastite.

\begin{tabular}{|c|c|c|c|c|c|c|}
\hline \multirow[t]{3}{*}{ Variáveis } & \multicolumn{6}{|c|}{ Mastite } \\
\hline & \multicolumn{2}{|c|}{ Sim } & \multicolumn{2}{|c|}{ Não } & \multirow[t]{2}{*}{ RP (IC95\%) } & \multirow[t]{2}{*}{$p$} \\
\hline & $\mathrm{n}$ & $\%$ & $n$ & $\%$ & & \\
\hline \multicolumn{7}{|c|}{$\begin{array}{l}\text { Iniciativa Hospital Amigo } \\
\text { da Criança }\end{array}$} \\
\hline Sim & 33 & 3,6 & 878 & 96,4 & $0,68(0,46-1,01)$ & 0,051 \\
\hline Não & 87 & 5,3 & 1.545 & 94,7 & & \\
\hline \multicolumn{7}{|c|}{$\begin{array}{l}\text { Orientação de aleitamento } \\
\text { materno no pré-natal }\end{array}$} \\
\hline Sim & 84 & 4,3 & 1.883 & 95,7 & $0,68(0,47-0,99)$ & 0,050 \\
\hline Não & 36 & 6,3 & 540 & 93,8 & & \\
\hline \multicolumn{7}{|c|}{$\begin{array}{l}\text { Orientação de aleitamento } \\
\text { materno e alojamento conjunto }\end{array}$} \\
\hline Sim & 81 & 4,2 & 1.849 & 95,8 & $0,67(0,45-0,96)$ & 0,030 \\
\hline Não & 39 & 6,4 & 574 & 93,6 & & \\
\hline \multicolumn{7}{|c|}{$\begin{array}{l}\text { Orientação de ordenha } \\
\text { e alojamento conjunto }\end{array}$} \\
\hline Sim & 70 & 4,5 & 1477 & 95,5 & $0,90(0,63-1,28)$ & 0,560 \\
\hline Não & 50 & 5,0 & 946 & 95,0 & & \\
\hline \multicolumn{7}{|c|}{ Fissura de mamilo } \\
\hline Sim & 77 & 9,2 & 758 & 90,8 & $0,27(0,19-0,39)$ & 0,000 \\
\hline Não & 43 & 2,5 & 1.665 & 97,5 & & \\
\hline \multicolumn{7}{|c|}{ Aleitamento } \\
\hline Sim & 66 & 3,8 & 1.681 & 96,2 & $0,56(0,39-0,79)$ & 0,001 \\
\hline Não & 54 & 6,8 & 742 & 93,2 & & \\
\hline \multicolumn{7}{|c|}{ Aleitamento exclusivo* } \\
\hline Sim & 22 & 4,2 & 500 & 95,8 & $0,47(0,27-0,83)$ & 0,009 \\
\hline Não & 22 & 8,9 & 224 & 91,1 & & \\
\hline
\end{tabular}

* Foi avaliado nos menores de seis meses.

Razão de prevalência bruta e ajustada e respectivos intervalos de confiança a $95 \%$ da associação entre a Iniciativa Hospital Amigo da Criança e a mastite $(n=2.543)$.

\begin{tabular}{lcccc}
\hline & RP $_{\text {bruta }}$ & IC95\% & RP $_{\text {ajustada }}$ & IC95\% \\
\hline Iniciativa Hospital Amigo da Criança & 0,68 & $0,46-1,01$ & 0,71 & $0,48-1,06$ \\
Fissura & 0,27 & $0,19-0,39$ & 0,27 & $0,19-0,40$ \\
Trabalho fora do lar & 0,64 & $0,44-0,92$ & 0,64 & $0,44-0,94$ \\
\hline
\end{tabular}


cente do conhecimento científico estabelecido sobre a mastite lactacional, por meio de consultas às bases de dados da PubMed e MEDLINE/ OVID, utilizando-se o filtro mastite, foram identificadas 612 referências sobre o tema, e nenhuma publicação que informasse ter sido investigada, especificamente, a associação entre a IHAC e a mastite. Do mesmo modo, na última revisão sistemática realizada pela OMS 1 sobre as causas e o manejo da mastite, não foram citados estudos que tivessem pesquisado a referida associação.

Sabe-se, no entanto, que as principais causas de mastite são a estase lática e a infecção, sendo, habitualmente, a estase a causa primária 1. Dentre as causas de estase do leite, incluem-se pega inadequada 14 , sucção ineficiente, esvaziamento incompleto da mama, restrição da freqüência ou duração das mamadas 14,22,23. Por conseguinte, esses problemas podem ser evitados, desde que eficientes medidas de intervenção sejam realizadas, precocemente, nas situações relacionadas à estase do leite, por meio de técnicas corretas de amamentação; rotinas essas recomendadas nos hospitais credenciados como amigos da criança.

Dentre as práticas da IHAC que favorecem o estabelecimento da amamentação, destacam-se a permanência da criança em alojamento conjunto, com orientação quanto às condutas maternas relacionadas com o manejo da amamentação, incluindo a orientação da pega e a posição adequada para amamentar; a amamentação sob livre demanda; a recomendação de não usar bicos e mamadeiras e suplementos alimentares indiscriminadamente, sem recomendação médica, além de ensinamentos sistemáticos às mães sobre a técnica de ordenhar o próprio leite no caso de superprodução e em casos em que elas necessitem afastar-se de seus filhos para trabalhar 18 .

Flores \& Filteau 8, conduzindo um estudo de intervenção, observaram que uma simples sessão de aconselhamento sobre amamentação após o parto, a importância do colostro, aleitamento exclusivo, amamentação sob livre demanda, pega e posição para amamentar esteve associada, um mês após o parto, a níveis significantemente menores de sódio-potássio no leite materno do grupo de intervenção, quando comparado ao grupo controle. Recente estudo de coorte conduzido por Schwartz et al. 24 revelou que assistência e aconselhamento às lactantes podem prevenir a instalação da mastite, e que essa patologia esteve associada ao desmame nas primeiras três semanas após o parto.

Em relação à ocorrência da mastite observada neste estudo, dados semelhantes têm si- do encontrados em outras pesquisas; apesar das variações de incidência entre os trabalhos publicados, a maioria informa uma incidência menor que $10 \% 1$. Essas variações foram atribuídas às limitações metodológicas observadas na maioria das pesquisas, tais como: amostras não representativas e populações selecionadas 1,17 . A pesquisa que relata a maior incidência de mastite (33\%) 13 inclui casos diagnosticados pela própria paciente e a amostra não foi considerada representativa para a população ${ }^{1}$. Mais recentemente, num estudo prospectivo com 946 mulheres americanas, observou-se que $9,5 \%$ delas tiveram, pelo menos, um episódio de mastite nos três primeiros meses após o parto 15. Em outro estudo de coorte, com seguimento de 1.075 mulheres australianas por telefone, $20 \%$ informaram que haviam tido mastite, sendo $75 \%$ dos casos nas sete primeiras semanas após o parto 25.

Um passo importante dos hospitais com o programa, que pode ter contribuído para a menor prevalência da mastite naquelas mulheres cuidadas durante o parto, foi o fato de as mulheres assistidas nesses serviços terem seu acompanhamento assegurado após a alta hospitalar, uma vez que a mastite ocorre mais comumente nas primeiras semanas do puerpério e decresce ao longo da lactação 1,17.

Nos dois hospitais do Município de Feira de Santana, credenciados como Amigos da Criança, as mães têm esse acompanhamento firmado por seis meses, com agendamento da primeira consulta para o ambulatório de incentivo ao aleitamento materno, na alta hospitalar. É possível que tenha ocorrido detecção e intervenção precoce nos fatores determinantes da mastite. Em estudo anterior, realizado em Feira de Santana, foram encontrados resultados semelhantes: dentre as 70 lactantes atendidas com mastite, $79 \%$ dos casos ocorreram nas primeiras oito semanas após o parto, e $63 \%$ dos partos foram realizados nos hospitais sem o programa 11 . Vale a pena ressaltar que, apesar de a mastite ser mais freqüente no período puerperal, pode ocorrer durante toda a lactação 1,11,25; por conseguinte, preferimos o termo mastite lactacional, ao invés de mastite puerperal.

No presente estudo, foi observada, também, associação entre a mastite e a fissura de mamilos. Existe um consenso de que dor e fissura de mamilos são fatores predisponentes para a mastite lactacional, mas não como causa necessária e suficiente 17 . Recente pesquisa, realizada por Foxman et al. 15, reforça essa idéia de que a mastite esteve associada à fissura de mamilo $\mathrm{e}$ ao uso de cremes antifúngicos. É importante lembrar que a fissura de mamilos pode ser uma 
porta de entrada para bactérias 15,26 pelos ductos lactíferos ou pelos linfáticos peri-ductais 11 . A partir de pequenas lesões na pele e rompimento da primeira barreira de defesa, as bactérias, sobretudo o Staphylococcus aureus, encontram condições apropriadas para o seu crescimento 11. Por outro lado, a causa mais comum de dor e trauma no mamilo é a pega inadequada 1,27 , que, por sua vez, pode levar a mãe a evitar alimentar o seu filho no peito e, assim, predispor-se à estase do leite 14 . A atenção à pega correta e o cuidado com as lesões do mamilo poderão evitar que o processo evolua para a mastite.

Ausentar-se do lar para trabalhar foi um fator que esteve associado a uma maior prevalência da mastite lactacional. Estudo retrospectivo, conduzido por Kaufmann \& Foxman 28, verificou que o trabalho materno, em tempo integral e fora do lar, esteve associado a um aumento de incidência da mastite, explicado, por esses autores, pela falta de tempo das mães para o esvaziamento completo da mama e por aumentar o tempo entre as mamadas.

Uma situação observada na análise bivariada e que requer atenção foi a amamentação ter se revelado como um fator de proteção contra a mastite na população estudada. A associação entre a freqüência das mamadas e a mastite lactacional não está suficientemente clara. Alguns autores sugerem que a mastite pode ocorrer, com maior freqüência, em mães com grande produção de leite, quando a mama não for suficientemente esvaziada 26 . Nesses casos, a maior freqüência das mamadas foi identificada como fator de proteção em relação à mastite 1,14 . WHO 1 ressalta que a introdução de outros alimentos, além do leite humano, nos primeiros seis meses de vida representa um fator de risco para a ocorrência de mastite, pois diminui o número de mamadas no peito. Por outro lado, Foxman et al. 15, estudando 926 lactantes por meio de uma coorte prospectiva, observaram que mamadas curtas e freqüentes estiveram associadas à mastite, mais do que as mamadas mais longas e menos freqüentes.

Não existe um consenso com relação a algumas variáveis apontadas por alguns autores como fatores predisponentes para a mastite lactacional e que ainda necessitam de maiores investigações, como uso de chupeta, prematuridade, peso ao nascer, primiparidade e ser mãe ainda jovem ${ }^{1}$. Na população estudada, esses fatores não estiveram associados, de modo significante, à mastite lactacional. Do mesmo modo, não foi observada relação entre a escolaridade materna e a mastite, evento que tem figurado como fator de risco para a mastite, em pesquisas anteriores 11,16, e, entretanto, não confirmado em estudos mais recentes 15. Segundo a WHO 1, esses fatores têm menor importância quando comparados aos relacionados à técnica da amamentação.

Os resultados do estudo sugerem que o programa desenvolvido pelos hospitais Amigos da Criança da Cidade de Feira de Santana, teve efeito preventivo em relação à mastite, evidenciado pela menor prevalência da mastite lactacional nas mulheres atendidas pelos hospitais com o programa.

Esse estudo reforça, por conseguinte, a relevância da implantação da IHAC nas maternidades, que, certamente, por seguirem os dez passos para o sucesso do aleitamento materno, incorporam, em suas rotinas, medidas que previnem a mastite lactacional, essa importante causa de desmame precoce, a qual interfere, sobretudo, na qualidade de vida da mulher-mãenutriz.

\section{Resumo}

O objetivo desse estudo foi estimar se o acompanhamento das lactantes nos hospitais credenciados como Amigos da Criança demonstrou ser fator de proteção contra a mastite no Município de Feira de Santana, Bahia, Brasil. Trata-se de um estudo transversal, com avaliação de 2.543 mães de crianças menores de um ano, entrevistadas por 104 universitários, em unidades de vacinação, selecionadas por estratificação simples. Foram calculadas medidas de associação (razão de prevalência, com IC95\%) e de significância estatística, através do teste do qui-quadrado com respectivo valor de p. Realizou-se análise multivariada através do modelo de regressão logística. Foi observada preva- lência significantemente menor de mastite entre as mulheres que pariram nos hospitais com o programa (3,6\% versus 5,3\%) ( $R P=0,68$; IC95\%: 0,46-1,01). As variáveis associadas a menores probabilidades de mastite foram: nascimento em hospitais com o programa ( $R P=0,71$; IC95\%:0,48-1,06), ausência de fissura mamilar $(R P=0,27$;C95\%:0,19-0,40) e ausência de trabalho materno fora do lar $(R P=0,64$; IC95\%:0,440,94). Notou-se, assim, efeito protetor contra a mastite nas mulheres atendidas nos hospitais com o programa.

Aleitamento Materno; Mastite; Avaliação de Programas; Prática em Hospital 


\section{Colaboradores}

G. O. Vieira e L. R. Silva tiveram substancial contribuição nas etapas de elaboração do projeto, desenho do estudo, aquisição dos dados, análise e interpretação dos resultados. C. M. C. Mendes teve relevante contribuição na análise bivariada, multivariada e interpretação dos dados. T. O. Vieira participou ativamente da revisão de literatura, coleta dos dados, digitação e análise descritiva e bivariada do estudo.

\section{Referências}

1. World Health Organization. Mastitis causes and management. Geneva: World Health Organization; 2000.

2. Fetherston C. Characteristics of lactation mastitis in a Western Australian cohort. Breastfeed Rev 1997; 5:5-11.

3. Semba RD. Mastitis and transmission of human immunodeficiency virus through breast milk. Ann N Y Acad Sci 2000; 918:156-62.

4. Willumsen JF, Filteau SM, Coustsoudis A, Uebel KE, Newell ML, Tomkins AM. Subclinical mastitis as a risk factor for mother-infant HIV transmission. Adv Exp Med Biol 2000; 478:211-23.

5. Willumsen JF, Filteau SM, Coutsoudis A, Newell ML, Rollins NC, Coovadia HM, et al. Breastmilk RNA viral load in HIV-infected South African woman: effects of subclinical mastitis and infant feeding. AIDS 2003; 17:407-14.

6. Michie C, Lockie F, Lynn W. The challenge of mastitis. Arch Dis Child 2003; 88:818-21.

7. Fetherston C. Mastitis in lactating women: physiology or pathology? Breastfeed Rev 2001; 9:5-12.

8. Flores M, Filteau S. Effect of lactation counselling on subclinical mastitis among Bangladeshi women. Ann Trop Paediatr 2002; 22:85-8.

9. Schneider HP, Raber G. Stillen, Stillschwierigkeiten und entzundliche Erkrankungen der weiblichen Brust. Ther Umsch 1993; 50:280-5.

10. Marchant DJ. Inflammation of the breast. Obstet Gynecol Clin North Am 2002; 29:89-102.

11. Sales AN, Vieira GO, Moura MSQ, Almeida SPTMA, Vieira TO. Mastite puerperal: estudo de fatores predisponentes. Rev Bras Ginecol Obstet 2000; 22:627-32.

12. Foxman B, Schwartz K, Looman SJ. Breastfeeding practices and lactation mastitis. Soc Sci Med 1994; 38:755-61.

13. Riordan JM, Nichols FH. A descriptive study of lactation mastitis in long-term breastfeeding women. J Hum Lact 1990; 6:53-8.

14. Fetherston C. Risk factors for lactation mastitis. J Hum Lact 1998; 14:101-9.

15. Foxman B, D'Arcy H, Gillespie B, Bobo JK, Schwartz K. Lactation mastitis: occurrence and medical management among 946 breastfeeding women in the United States. Am J Epidemiol 2002; 155:10314.

16. Kinlay JR, O'Connell DL, Kinlay S. Risk factors for mastitis in breastfeeding women: result of prospec- tive cohort study. Aust N Z J Public Health 2001; 25:115-20.

17. Barbosa-Cesnik C, Schwartz K, Foxman B. Lactation mastitis. JAMA 2003; 289:1609-12.

18. Organização Mundial da Saúde. Evidências científicas dos dez passos para o sucesso do aleitamento materno. Brasília: Organização Pan-Americana da Saúde/Ministério da Saúde; 2001.

19. Núcleo de Informações e Pesquisas Econômicas e Sociais de Feira de Santana. Dados gerais sobre Feira de Santana. http://www.nipes.feiradesantana.ba.gov.br/f_dados.htm (acessado em 13/Jul/ 2004).

20. Departamento de Informação e Informática do SUS. Informações de saúde. http://tabnet.datasus.gov.br/cgi/tabcgi.exe?ibge/cnv/popba.def (acessado em 22/Fev/2005).

21. Zhang J, Yu KF. What's the relative risk? A method of correcting the odds ratio in cohort studies of common outcomes. JAMA 1998; 280:1690-1.

22. Lawrence RA. Cuidados generales de la pareja madre-hijo durante la lactancia In: Lawrence RA, organizador. La lactancia materna: Una guia para la profesión médica. Madrid: Mosby; 1996. p. 22186.

23. Riordan J, Auerbach KG. Breastfeeding and human lactation. Sudbury: Jones and Bartlett; 1999.

24. Schwartz K, D'Árcy HJ, Gillespie B, Bobo J, Longeway M, Foxman B. Factors associated with weaning in the first 3 months postpartum. J Fam Pract 2002; 51:439-44.

25. Kinlay JR, O' Connell DL, Kinlay S. Incidence of mastitis in breastfeeding women during the six months after delivery: a prospective cohort study. Med J Aust 1998; 169:310-2.

26. Vogel A, Hutchinson BL, Mitchell EA. Mastitis in the first year postpartum. Birth 1999; 26:218-25.

27. Centuori S, Burmaz T, Ronfani L, Fragiacomo M, Quintero S, Pavan R, et al. Nipple care, sore nipples and breastfeeding: a randomized trial. J Hum Lact 1999; 15:127-32.

28. Kaufmann R, Foxman B. Mastitis among lactating women. Occurrence and risk factors. Soc Sci Med 1991; 33:701-5.

Recebido em 24/Ago/2004

Versão final reapresentada em 25/Jul/2005

Aprovado em 14/Out/2005 